\title{
Effect of surface roughness on grain growth and sintering of alumina
}

\author{
PADMAJA PARAMESWARAN NAMPI ${ }^{\dagger, *}$, SHOICHI KUME, YUJI HOTTA and \\ KOJI WATARI \\ Advanced Sintering Technology Group, Advanced Manufacturing Research Institute, National Institute of \\ Advanced Industrial Science and Technology (AIST), 2266-98 Anagahora, Shimoshidami, Moriyama-ku, \\ Nagoya 463-8560, Japan \\ ${ }^{\dagger}$ Present Address: Bioceramics Laboratory, Biomedical Technology Wing, Sree Chitra Tirunal Institute for \\ Medical Sciences and Technology, Thiruvananthapuram 695 012, India
}

MS received 19 August 2009; revised 12 September 2009

\begin{abstract}
The production of ceramic bodies with less surface roughness is industrially important when one considers the aspect of final machining processes. Hence an attempt have been made to study the variation in surface roughness parameters $\left(R_{\mathrm{a}}, R_{\mathrm{y}}, R_{\mathrm{z}}\right)$ of alumina having three different kinds of roughness features at different sintering temperatures. Variation in surface roughness properties are also correlated with grain size. $R_{\mathrm{z}}$ shows significant difference between fine and intermediate surfaces, hence predicts small difference in their microstructural features. As a general trend, average grain size increases with increase in sintering temperature, but wide distribution of grains with enhanced non-uniform grain growth is observed when the surface is coarse. Hence, creation of fine surface in the green body is necessary for homogeneously distributed grains with controlled uniform grain growth. The final roughness and grain size of the sintered alumina depend on the initial surface roughness of the green body.
\end{abstract}

Keywords. $\quad \mathrm{Al}_{2} \mathrm{O}_{3}$; electron microscopy; grain growth; sintering; surface roughness.

\section{Introduction}

Making ceramic shapes with perfect size, flatness and roughness seeks high importance when one considers the quality of the final product. Ceramic material shrinks linearly around $20 \%$ during sintering. In general, sintered ceramic product having accurate size and shape with perfect flatness and little roughness is difficult to produce since the sintering results in inhomogeneous shrinkage and microstructure. Hence, usually machining-grinding and polishing is required for making the final ceramic product with accurate size and shape.

However, these machining inevitably introduce flaws in the form of surface-breaking cracks (Franco et al 1997). It is also understood that the surface roughness increases when machining processes has been executed due to the presence of formation of large pores at the inner parts of the products (private communication). It has been well documented that grinding often generates sub-surface damage and deleterious effects on strength and performance of ceramic components (Hockey and Rice 1979; Marshall et al 1983; Kirchner 1984; Jahanmir 1993).

*Author for correspondence (padmavasudev@gmail.com; padmaja@sctimst.ac.in)
It has also been reported that surface roughness is one of the major parameters which influences properties including toughness (Boccaccini and Winkler 2002), crack deflection (Boccaccini and Winkler 2002), flexural strength and friction (Wong et al 1998; Bhamra et al 2002), indentation hardness (Narayan and Hancock 2003), elastic modulus (Narayan, and Hancock 2003), and brittle fracture index (Narayan and Hancock 2003). In addition to avoiding the above-mentioned defects such as cracks, flaws and pores which are important for retaining high performance of the component, reduction in processing steps and processing time also seeks high importance, for mass production with reduced manufacturing cost.

Therefore, there is high necessity for the production of ceramic without final machining. In this case, evaluation of surface roughness by variation in sintering temperature conditions is important since final roughness of the sintered material might depend on the initial roughness induced during forming of the green compact.

However, only limited literature is available regarding change in surface roughness with sintering without final machining. Hence, attempts have been made to investigate the change in surface roughness parameters of alumina pellets with different surface roughness at three different sintering temperatures and correlation with microstructural features have been tried. 


\section{Experimental}

$\alpha$-Alumina powder (Grade-TMDAR, Taimei Chemicals Co. Ltd., Japan, purity: $99.99 \%$, mean particle size: $0.16 \mu \mathrm{m}$ ) was used as the raw material. The surface texture has been modified by pressing the alumina powder with micro-configured die faces. The fine die surface $\left(R_{\mathrm{a}}\right.$ : $0.1 \mu \mathrm{m}, R_{\mathrm{z}}: 0.7 \mu \mathrm{m}, R_{\mathrm{y}}: 1.0 \mu \mathrm{m}$, represented as 'fine') was created by polishing the die surface using sandpaper $\# 1000$, while the intermediate roughness $\left(R_{\mathrm{a}}: 0.5 \mu \mathrm{m}, R_{\mathrm{z}}\right.$ : $5 \mu \mathrm{m}, R_{\mathrm{y}}: 7 \mu \mathrm{m}$, represented as 'intermediate') was made by using that of \#400. The coarse die surface $\left(R_{\mathrm{a}}: 2 \mu \mathrm{m}\right.$, $R_{\mathrm{z}}: 13 \mu \mathrm{m}, R_{\mathrm{y}}: 15 \mu \mathrm{m}$, represented as 'coarse') was obtained by machining using a fraise device. The alumina powders were consolidated into compacts by pressing uniaxially at $30 \mathrm{MPa}$ using dies with above mentioned surface parameter values. The pellets were calcined at $800^{\circ} \mathrm{C}$ and sintered at 1350,1500 and $1600^{\circ} \mathrm{C}$ for $2 \mathrm{~h}$. The surface roughness in an area of $2 \times 2 \mathrm{~mm}$ of the samples has been measured using a Laser three dimensional Surface Roughness Analyser (COMS, EMS2002AD-3D 100XY, Japan). The surface scanning of the sample specimen was done using the laser scanner (Keyence Corporation, Japan). The roughness parameters, the surface roughness image as well as the profiles were obtained using EMS2002AD-3D program. The $R_{\mathrm{a}}, R_{\mathrm{z}}$ and $R_{\mathrm{y}}$ values of each surface were obtained by taking the average of five profiles. The microstructures of the sintered samples have been observed by using a Scanning Electron Microscope (SEM: JEOL, JSM 5600 LV, Japan). The average grain sizes were calculated from the microstructures using the linear-intercept technique (Gadelmawla et al 2002).

\section{Results and discussion}

\subsection{Surface roughness}

The variation in $R_{\mathrm{a}}, R_{\mathrm{z}}$ and $R_{\mathrm{y}}$ of green samples, sample calcined at $800^{\circ} \mathrm{C}$ and as-sintered surfaces (sintering temperatures of 1350,1500 and $1600^{\circ} \mathrm{C}$ ) of alumina pellets with 'fine' intermediate' and 'coarse' surfaces have been presented in figures $1-3$. The variation in surface roughness has been studied by analyzing the variation in surface roughness parameters $R_{\mathrm{a}}, R_{\mathrm{z}}$ and $R_{\mathrm{y}}$ of various surfaces at different temperatures. $R_{\mathrm{a}}$ is defined as the average absolute deviation of the roughness irregularities from the mean line over one sampling length. It is also defined as the summation of negative and positive deviations from a 'mean plane' fit over the surface of interest. $R_{\mathrm{z}}$ is the ten-point height, which is defined by the international ISO system as the difference in height between the average of the five highest peak and five lowest valleys along the assessment length of the profile and $R_{\mathrm{y}}$ is defined as the largest peak to valley height (Mendelson 1969).
The variation in $R_{\mathrm{a}}$ value with temperature for samples obtained by consolidation using fine, intermediate and coarse punches is shown in figure 1. $R_{\mathrm{a}}$ values of $0.66 \mu \mathrm{m}$, $0.72 \mu \mathrm{m}$ and $2.4 \mu \mathrm{m}$ are observed for green samples, when fine, intermediate and coarse punches are used for pelletization. There is least difference in $R_{\mathrm{a}}$ for samples at $150^{\circ} \mathrm{C}$ and $800^{\circ} \mathrm{C}$ calcined samples. As the temperature is increased from $800^{\circ} \mathrm{C}$ to $1350^{\circ} \mathrm{C}$, the $R_{\mathrm{a}}$ value decreases. This may be due to the effective migration of surface grains, which lessens the roughness during initial stages of the process of sintering. $R_{\mathrm{a}}$ value of $0.46 \mu \mathrm{m}$ has been observed for the pellet with fine surface, sintered at $1350^{\circ} \mathrm{C}$. The value increases to $0.47 \mu \mathrm{m}$ and $0.49 \mu \mathrm{m}$ as the sintering temperature is increased to $1500^{\circ} \mathrm{C}$ and $1600^{\circ} \mathrm{C}$, respectively which indicates there is least difference

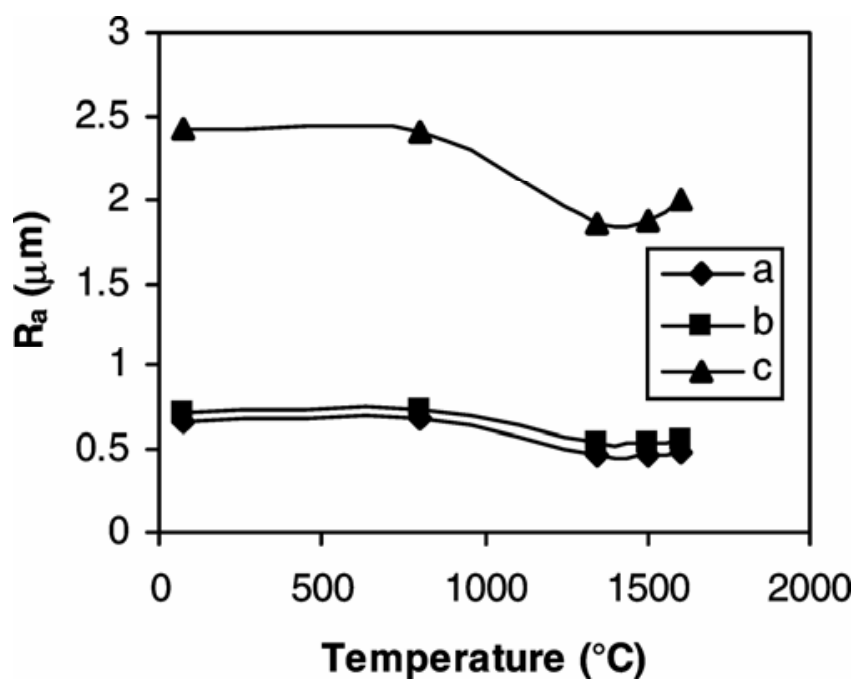

Figure 1. Variation in $R_{\mathrm{a}}$ with temperature: (a) fine, (b) intermediate and (c) coarse.

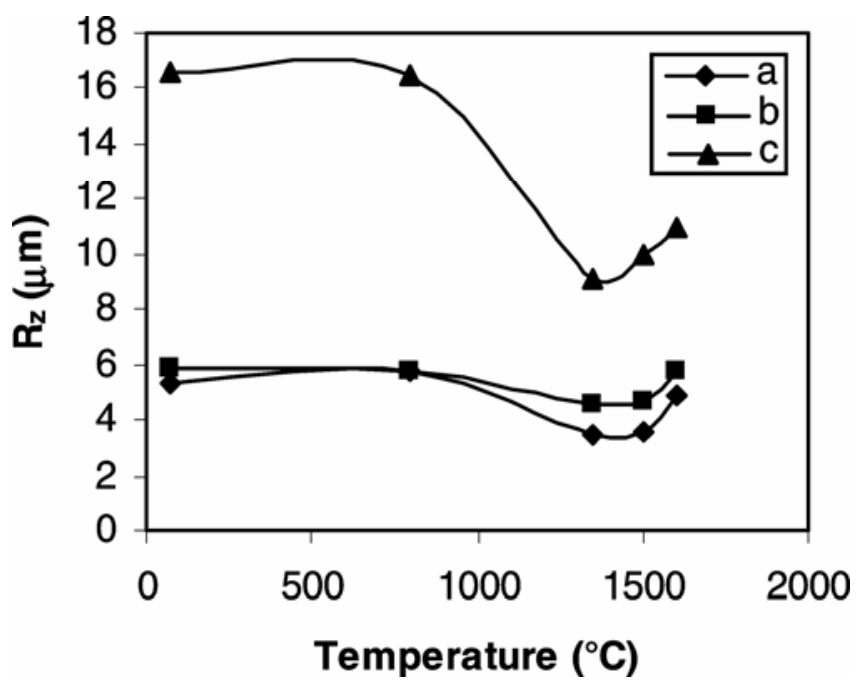

Figure 2. Variation in $R_{\mathrm{z}}$ with temperature (a) fine, (b) intermediate and (c) coarse. 
in $R_{\mathrm{a}}$ as the sintering temperature is increased. The surface roughness value increases to $0.54 \mu \mathrm{m}$ when a punch of intermediate roughness is used for consolidation and the increase in sintering temperature had little effect in the variation of $R_{\mathrm{a}}$ value. On the other hand, the $R_{\mathrm{a}}$ value increases to $1.85 \mu \mathrm{m}$ which increases to $2 \mu \mathrm{m}$ as the sintering temperature is increased from $1350^{\circ} \mathrm{C}$ to $1600^{\circ} \mathrm{C}$ when the surface is very coarse.

The variations in $R_{\mathrm{z}}$ and $R_{\mathrm{y}}$ are also taken into consideration since they give much information regarding the roughness in a larger scale. $R_{\mathrm{z}}$ and $R_{\mathrm{y}}$ are also affected by the microstructure; $R_{\mathrm{z}}$ and $R_{\mathrm{y}}$ values vary considerably due to the presence of pores, and/or protuberances, while $R_{\mathrm{a}}$ values are less affected by minor defects since $R_{\mathrm{a}}$ is the total average roughness.

The variation of $R_{\mathrm{z}}$ with temperature is presented in figure 2. Least difference in $R_{\mathrm{z}}$ is observed for samples at $150^{\circ} \mathrm{C}$ and samples calcined at $800^{\circ} \mathrm{C}$. For the fine surface, $R_{\mathrm{z}}$ value shows variation from 3.4 to $3.9 \mu \mathrm{m}$ as the temperature is increased from 1350 to $1600^{\circ} \mathrm{C}$. The $R_{z}$ values change from 3.4 to $9 \cdot 1 \mu \mathrm{m}$ as the roughness is increased from fine to coarse at $1350^{\circ} \mathrm{C}$ and maximum value of $11.0 \mu \mathrm{m}$ is observed for the coarse surface at $1600^{\circ} \mathrm{C}$. It is also observed that there is a dramatic increase in $R_{\mathrm{z}}$ with the sintering temperature when a coarse punch is used for consolidation.

The variation in $R_{\mathrm{y}}$ with sintering temperature is presented in figure 3 . For green samples, $R_{\mathrm{y}}$ value of $6.06 \mu \mathrm{m}$, $6.5 \mu \mathrm{m}$ and $19.91 \mu \mathrm{m}$ is observed while consolidated using fine, intermediate and coarse punch, respectively. $R_{\mathrm{y}}$ of $4.4 \mu \mathrm{m}$ is observed for fine surface at $1350^{\circ} \mathrm{C}$ which changes to $4.5 \mu \mathrm{m}$ and $4.9 \mu \mathrm{m}$ as the sintering temperature is varied to $1500^{\circ} \mathrm{C}$ and $1600^{\circ} \mathrm{C}$, respectively. When an intermediate punch is used for pelletization, the $R_{\mathrm{y}}$ value is increased to $4.8 \mu \mathrm{m}$ at $1350^{\circ} \mathrm{C}$ and shows a maximum value of $6 \mu \mathrm{m}$ at $1600^{\circ} \mathrm{C}$. When the surface is

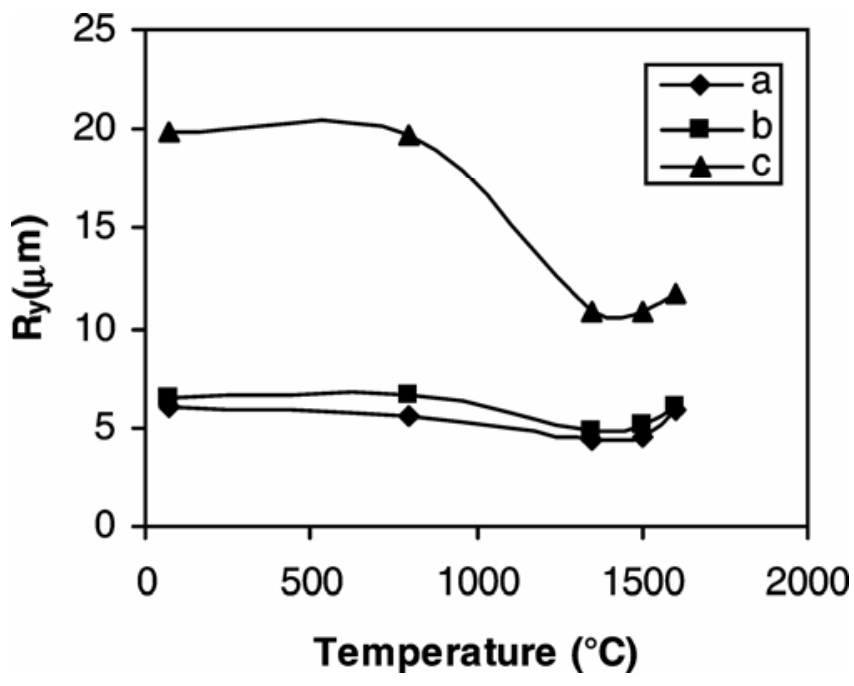

Figure 3. Variation in $R_{\mathrm{y}}$ with temperature: (a) fine, (b) intermediate and (c) coarse. coarse, a value of $10 \cdot 8 \mu \mathrm{m}$ is observed at $1350^{\circ} \mathrm{C}$, reaches a value of $11.8 \mu \mathrm{m}$ as the temperature is increased to $1600^{\circ} \mathrm{C}$. The variation in $R_{\mathrm{y}}$ with temperature also shows less variation in $R_{\mathrm{y}}$ value when the surface is fine.

The results show the importance of surface parameters, especially $R_{\mathrm{z}}$ in determining the surface properties.

\subsection{Surface microstructure}

The grain growth process is established by analysing the microstructural features. The microstructures of the assintered surfaces of the samples sintered at 1350, 1500 and $1600^{\circ} \mathrm{C}$ has been presented in figure 4 . At all sintering temperatures, the microstructure shows much homogeneous distribution of grains for the fine surface. Table 1 shows the average grain size values and their standard deviations of the sample pellets with fine, intermediate and coarse surfaces and sintered at $1350^{\circ} \mathrm{C}, 1500^{\circ} \mathrm{C}$ and $1600^{\circ} \mathrm{C}$. Lesser the surface roughness, smaller is the grain size. This is clearly evident from the microstructure of samples sintered at $1600^{\circ} \mathrm{C}$ as shown in figure 4 . Even if the usual typical sintering temperature of TMDARalumina is around $1350^{\circ} \mathrm{C}$, we are using the microstructures at higher temperatures for effective comparison of average grain size.

\subsection{Average grain size}

The variation in grain size with temperature is presented in figure 5. The uniformity and homogeneity of grain growth is also found to be more for the fine surface. For example, for the fine surface, the grain size ranges from $1.5 \mu \mathrm{m}$ to $11.5 \mu \mathrm{m}$ as the temperature is increased from $1350^{\circ} \mathrm{C}$ to $1600^{\circ} \mathrm{C}$, while the average grain size ranges from $2.5 \mu \mathrm{m}$ to $18.2 \mu \mathrm{m}$ indicating increased grain growth rate for the coarse surface in the same range of temperature. As a general trend, the grain size is also found to increase as the temperature is increased, irrespective of surface roughness.

The variation in $R_{\mathrm{a}}, R_{\mathrm{z}}$ and $R_{\mathrm{y}}$ with average grain size is presented in figures $6-8$, respectively. Least difference in $R_{\mathrm{a}}$ is observed for fine or intermediate roughness surface, especially at lower temperature. High $R_{\mathrm{a}}$ value is observed for the coarse surface at all sintering temperatures. While considering $R_{\mathrm{z}}$, the value shows prominent difference for fine and intermediate surface, even at low sintering temperatures. Hence $R_{\mathrm{z}}$ shows even the small difference in microstructural features between fine and intermediate, which is less observable when $R_{\mathrm{a}}$ or $R_{\mathrm{y}}$ is considered, since $R_{\mathrm{a}}$ is the total mean roughness and $R_{\mathrm{y}}$ is the maximum peak to valley height in the surface profile. A significant increase in $R_{\mathrm{z}}$ is observed for a coarse surface which indicates the rapid grain growth rate as the temperature is increased from $1350^{\circ} \mathrm{C}$ to $1500^{\circ} \mathrm{C}$. This is also in accordance with the microstructural results. Variation in $R_{\mathrm{y}}$ shows the same trend as that of $R_{\mathrm{a}}$. 
Table 1. Variation in average grain size with punch roughness at different sintering temperatures.

\begin{tabular}{lccc}
\hline & \multicolumn{2}{c}{ Average grain size with standard deviation $(\mu \mathrm{m})$} \\
\cline { 2 - 4 } Temperature $\left({ }^{\circ} \mathrm{C}\right)$ & \multicolumn{1}{c}{ Fine } & Intermediate & Coarse \\
\hline 1350 & $1 \cdot 5 \pm 0 \cdot 18$ & $1 \cdot 7 \pm 0.2$ & $2 \cdot 5 \pm 0.64$ \\
1500 & $4 \cdot 8 \pm 0.27$ & $5.9 \pm 0.92$ & $8.5 \pm 0.9$ \\
1600 & $11.5 \pm 1.09$ & $13 \cdot 2 \pm 1.03$ & $18.2 \pm 1 \cdot 1$ \\
\hline
\end{tabular}

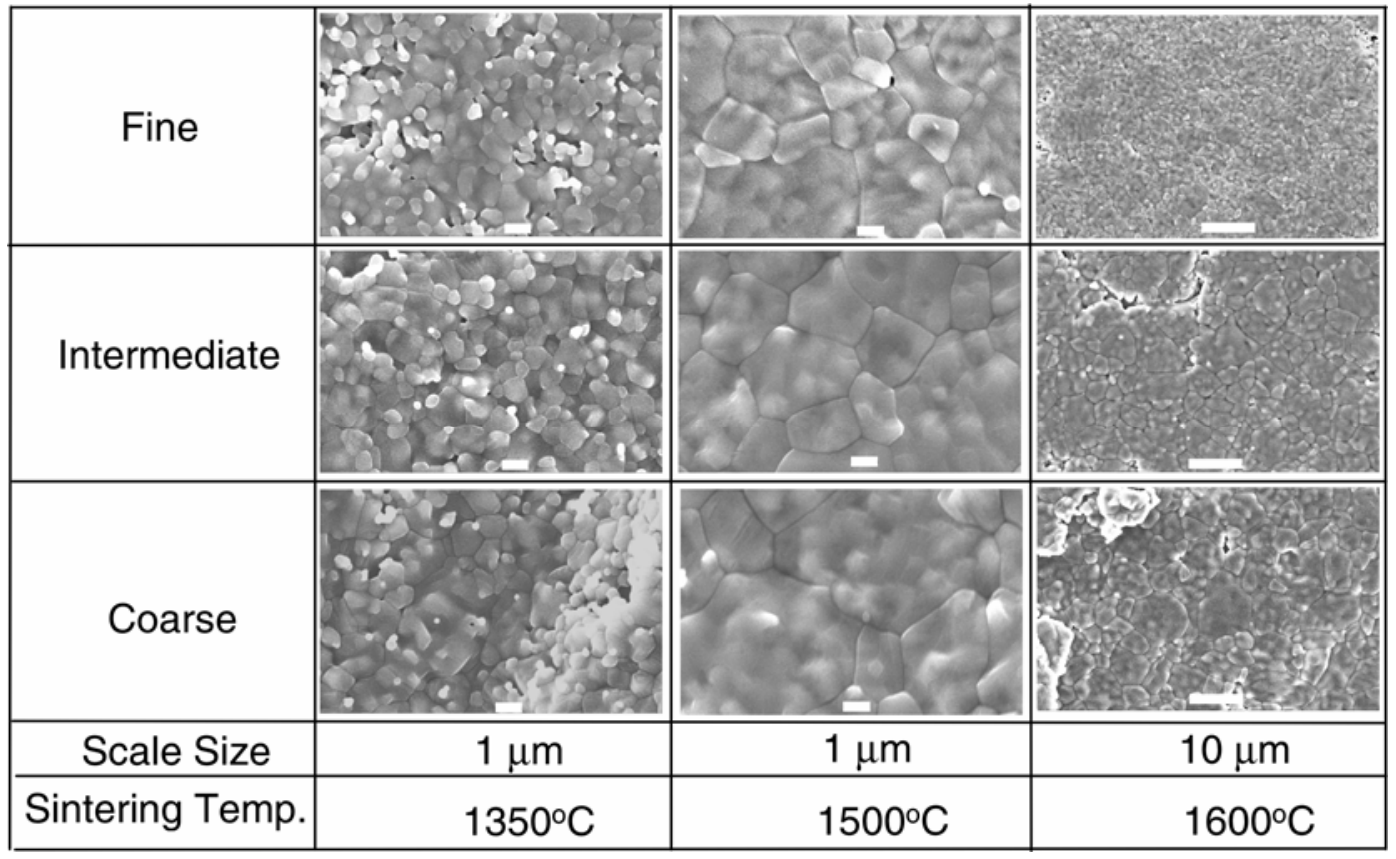

Figure 4. Microstructures of as-sintered samples.

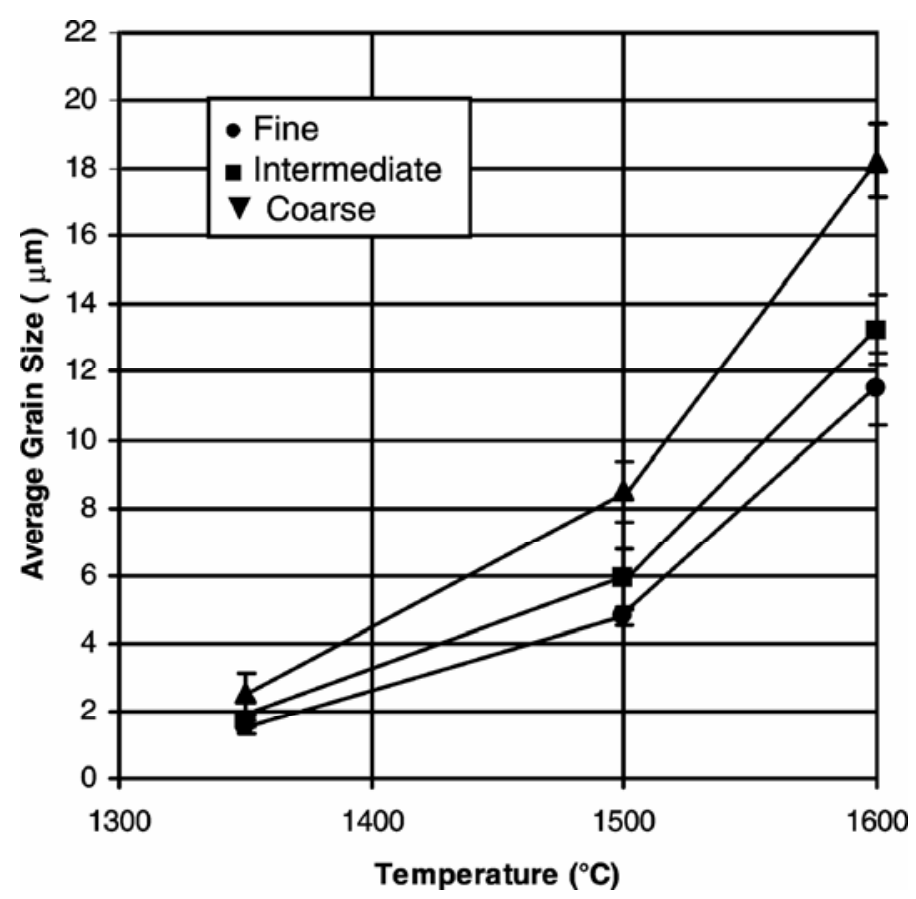

Figure 5. Variation in average grain size with sintering temperature. 
Figure 9 indicates the SEM of polished surfaces and inner portion of sample pelletized using fine, intermediate and coarse punch and sintered at $1600^{\circ} \mathrm{C}$. From the SEM of polished surface, we also conclude that the agglomerated-like grain we are observing in the as-sintered surface is a single grain. There is least difference in average grain size between the surface $(11.5 \mu \mathrm{m})$ and inner portion $(10.9 \mu \mathrm{m})$ of the pellet with fine surface, sintered at $1600^{\circ} \mathrm{C}$. An average grain size of $13.2 \mu \mathrm{m}$ is observed for the surface grain, whereas a grain size of $9.7 \mu \mathrm{m}$ is observed for the inner portion of sample pelletized using intermediate punch and sintered at $1600^{\circ} \mathrm{C}$. When pelletized using coarse punch, for the sample sintered at $1600^{\circ} \mathrm{C}$, an average grain size of $18.2 \mu \mathrm{m}$ is observed for the surface while an average grain size of $7.2 \mu \mathrm{m}$ is observed for interior portion. The larger grain size on the surface may be due to the possibility of enhanced diffusion on the surface during the process of grain growth (He and Ma 2000). The difference in grain size distribution between the surface and the interior portion is much pronounced when a coarse punch is used for pelletization.

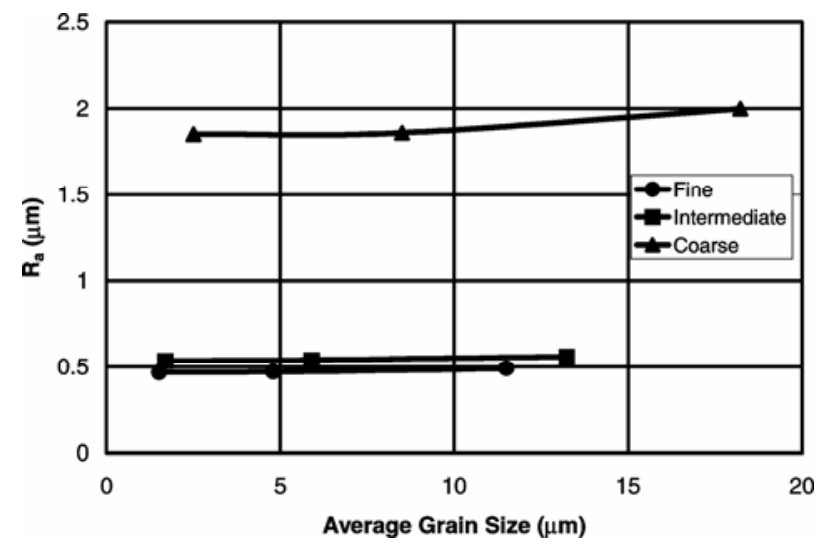

Figure 6. Variation in $R_{\mathrm{a}}$ with average grain size at different surface roughness

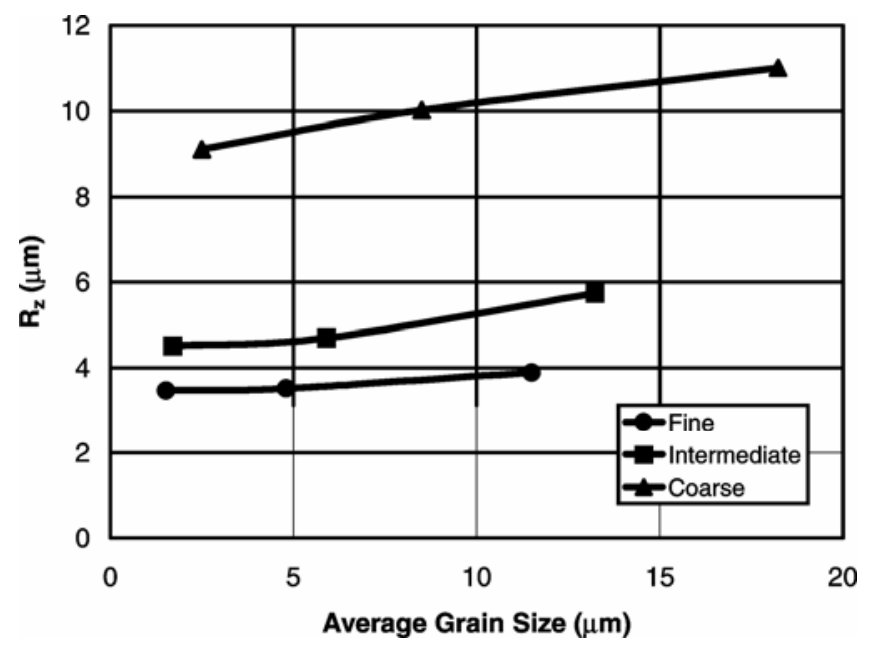

Figure 7. Variation in $R_{\mathrm{z}}$ with average grain size at different surface roughness.
For the compact with coarse surface, the surface area in more and more particles can come in contact, hence surface diffusion can be enhanced, but the deformation at the contact points may not be uniform due to the surface inhomogeneity. But this deformation may be uniform, for the fine surface, since regular stacked arrangement of particles causes uniform 'neck' formation and hence uniform grain growth is possible. It is found that the process during initial stage of grain growth determines the final sintered grain growth properties. Thus even at $1600^{\circ} \mathrm{C}$, non-uniform inhomogeneous grain growth is observed for the pellet consolidated using a coarse surface punch. It is observed that broader grain size distribution shows a greater driving force for grain growth. Hence for the coarse surface, grain growth is much enhanced compared to fine surface.

Thus the final roughness of the sinter and the process of grain growth are determined by the initial roughness created in the compact by using punches with different surface roughness. The driving force for the increase in grain size is the difference in energy between the particles with different curvatures. Thus, when the grain boundaries tend to form flat surfaces with less roughness, the driving force for boundary migration is eliminated, little grain growth occurs (Wong et al 1998).

Thus it is important to make compacts with less surface roughness in order to get homogeneous, uniform and relatively fine grains in the final sintered material.

\section{Conclusions}

Since the final roughness of the sintered compact depends on the initial roughness induced during forming of the green compact, attempts have been made to study the variation in roughness parameters at various sintering temperatures. It is important to make compacts with low

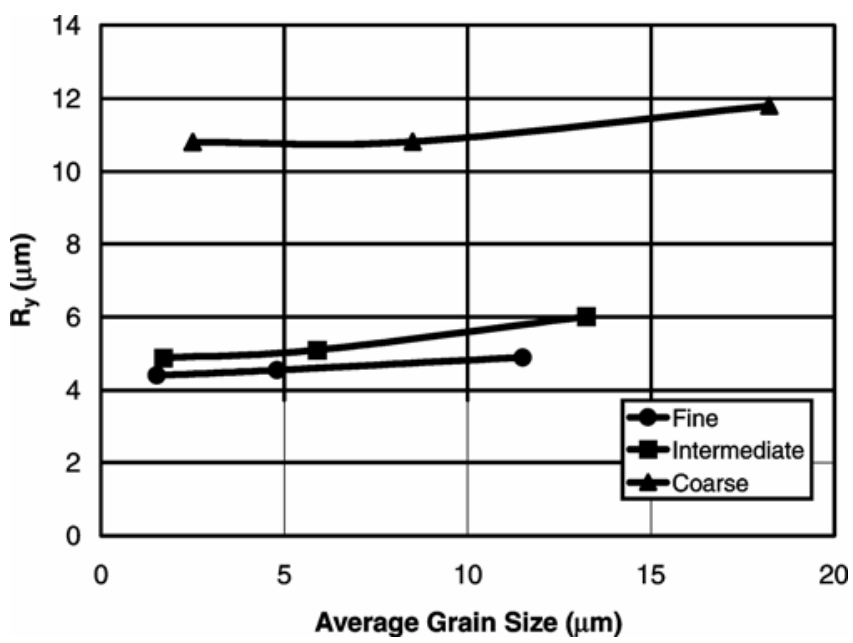

Figure 8. Variation in $R_{\mathrm{y}}$ with average grain size at different surface roughness. 


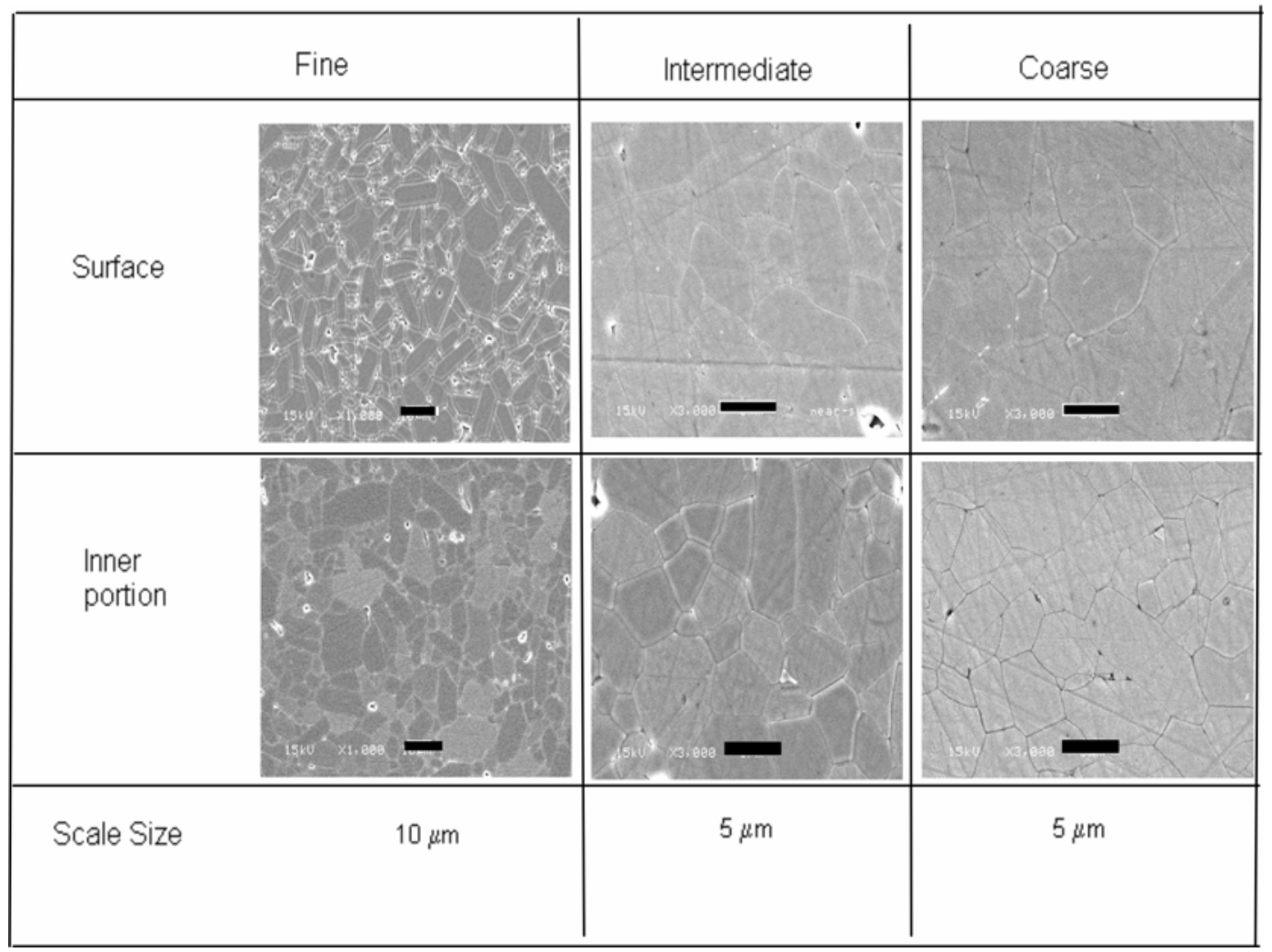

Figure 9. SEM micrographs of surface and inner portion of sample pelletized using fine, intermediate and coarse punch $\left(\right.$ sintered at $\left.1600^{\circ} \mathrm{C}\right)$.

surface roughness for having homogeneous uniform microstructure with inhibited grain growth. For the fine surface, the grain size ranges from $1.5 \mu \mathrm{m}$ to $11.5 \mu \mathrm{m}$ as the temperature is increased from 1350 to $1600^{\circ} \mathrm{C}$, while the average grain size ranges from $2.5 \mu \mathrm{m}$ to $18.2 \mu \mathrm{m}$ indicating increased grain growth rate for the coarse surface in the same range of temperature. $R_{\mathrm{z}}$ value shows significant difference between fine and intermediate surfaces, even at low sintering temperatures, hence predicts small difference in their microstructural features, which is less observable when $R_{\mathrm{a}}$ or $R_{\mathrm{y}}$ is considered. There is least difference in the average grain size between surface and inner portion when a fine surface punch is used for pelletization, while much larger surface grain size is observed, compared to the grain size of the inner portion when a coarse punch is used for consolidation.

\section{Acknowledgements}

One of the authors (PPN) is grateful to the National Institute of Advanced Industrial Science and Technology (AIST), Nagoya, Japan for a Post-doctoral Research
Fellowship. The technical assistance from K Nakamura is gratefully acknowledged.

\section{References}

Bhamra G, Palin W M and Fleming G J 2002 J. Dent. 30153 Boccaccini A R and Winkler V 2002 Composites Part A-Appl. Sci. \& Manufac. 33125

Franco A, Roberts, S G and Warren P D 1997 Acta Mat. 45 1009

Gadelmawla E S, Koura M M, Maksoud T M A, Elewa I M and Soliman H H 2002 J. Mater. Proc. Tech. 123133

He Z and Ma J 2000 Mater. Lett. 4414

Hockey B J and Rice R W (eds) 1979 The science of ceramic machining and surface finishing II, NBS SP 562 (Washington DC: US Government Printing Office)

Jahanmir S (ed.) 1993 Machining of advanced materials, NIST SP847 (Washington DC: US Government Printing Office)

Kirchner H P 1984 J. Am. Ceram. Soc. 67127

Marshall D B, Evans A G, Yakub B T K, Tien J W and Kino G S 1983 Proc. R. Soc. London $\mathbf{A 3 8 5} 461$

Mendelson M I 1969 J. Am. Ceram. Soc. 52443

Narayan P and Hancock B 2003 Mater. Sci. Eng. A355 24

Wong H C, Umehara N and Kato K 1998 Wear 218237 